\title{
Dependence of average path length betatron motion in a storage ring
}

\author{
Yoshihiko Shoji* \\ Laboratory of Advanced Science and Technology for Industry (LASTI), University of Hyogo, \\ 3-1-2, Koto, Kamigori-cho, Ako-gun, Hyogo, Japan \\ (Received 29 March 2005; published 27 September 2005)
}

\begin{abstract}
The dependence of path length on betatron motion in a storage ring is analytically calculated from equations of motion using curvilinear coordinates. It is demonstrated that the change of average path length for one revolution is expressed by a sum of the products of invariant of betatron motion and chromaticity in both the horizontal and vertical directions. Because of this effect, a quasi-isochronous electron storage ring should have a considerable momentum spread when the chromaticities are large.
\end{abstract}

DOI: 10.1103/PhysRevSTAB.8.094001

PACS numbers: 29.20.Dh, 29.27. $-\mathrm{a}, 41.75 .-\mathrm{i}$

\section{INTRODUCTION}

A quasi-isochronous (QI) electron synchrotron, which implies a very small momentum compaction factor $(\alpha)$, can store a very short electron bunch. When its bunch length is a few $\mathrm{mm}$ or less, an extremely strong coherent synchrotron radiation in the $\mathrm{THz}$ frequency region is emitted even with a low stored beam current [1]. This radiation has a continuous wavelength spectrum [2] and different applications from those with long wavelength free-electron laser. In the operation of QI ring, the shorter the bunch is, the broader the wavelength region is. The idea of the bunch shortening is derived from a well-known expression where the equilibrium bunch length is proportional to $\sqrt{\alpha}$. However, in extreme QI conditions, we must consider many effects that are negligible under normal conditions. One of them is bunch lengthening by a linear betatron motion [3], which is location dependent. In this article we sought to investigate the second order effect, the shift in average path length for one revolution by betatron motion. Henceforth, this betatron amplitude dependent shift is simply referred to as "the shift." A main consequence of the shift is a betatron amplitude dependent momentum shift.

The path length has a small dependence on the betatron oscillation amplitude. Wiedemann [4] already deduced an analytical formula for the shift, although his equation is insufficient since it does not contain important second order effects, such as sextupoles. Before that work Emery had given more accurate equation [5],

$$
\begin{aligned}
\Delta C= & -2 \pi\left(\xi_{X}^{\mathrm{QUAD}}+\xi_{X}^{\mathrm{SEXT}}\right) J_{X}-2 \pi\left(\xi_{Y}^{\mathrm{QUAD}}\right. \\
& \left.+\xi_{Y}^{\mathrm{SEXT}}\right) J_{Y} ;
\end{aligned}
$$

here $\Delta C$ is the shift of the average path length for one revolution, $\xi^{\mathrm{QUAD}}$ and $\xi^{\mathrm{SEXT}}$ are the chromaticity contributions of the quadrupoles and sextupoles and $J$ is the invariant of betatron motion, a half of Courant Snyder invariant. The suffix " $X$ " or " $Y$ " shows the directions of

*Email address: shoji@lasti.u-hyogo.ac.jp betatron motions. However Emery did not provide the derivation for Eq. (1). Later Forest showed that this surprisingly simple relation is a consequence of the simplecticity [6]. In the following section, we derive Eq. (1) starting from the general equations of motion using curvilinear coordinates, including the edge field effects. In the next section, the consequence of this effect is discussed and is calculated using the parameters of NewSUBARU, an existing QI storage ring.

\section{DEPENDENCE OF CIRCUMFERENCE ON BETATRON MORTION}

\section{A. Basic equations}

The coordinates in this report, $x, y$, and $s$, are the displacements from the reference electron in the radial and vertical directions and the azimuthal coordinate, respectively. The Twiss parameters are referred to as $\alpha(s)$, $\beta(s)$, and $\gamma(s)$, and the betatron phase as $\psi(s)$. The $\alpha$ without a suffix " $X$ " or " $Y$ " is the momentum compaction factor. In this report, we assume that there is not a skew magnetic element such as vertical curvature. Terms to $x$ and $y$ higher than second order are ignored.

Some books $[7,8]$ provide the equations of motion for a particle up to the second order as

$$
\begin{aligned}
x^{\prime \prime}+\left(h^{2}-k\right) x= & \left(2 h k-h^{3}+\frac{1}{2} m\right) x^{2} \\
& +\frac{1}{2}\left(h^{\prime \prime}-h k-m\right) y^{2}+h^{\prime}\left(x x^{\prime}+y y^{\prime}\right) \\
& +\frac{1}{2} h\left(x^{\prime 2}-y^{\prime 2}\right)+h \delta+\left(2 h^{2}-k\right) \delta \\
y^{\prime \prime}+k y= & -(m+2 h k) x y+h^{\prime}\left(x y^{\prime}-x^{\prime} y\right)+h x^{\prime} y^{\prime}+k \delta .
\end{aligned}
$$

The prime (') indicates the derivative to $s$ and $\delta$ is the relative momentum displacement. The $h(s), k(s)$, and $m(s)$ are the inverse of the curvature of radius of the reference orbit, the quadrupole field component, and the sextupole field component, respectively. They are 


$$
\begin{aligned}
h(s) & =1 / \rho(s), \\
k & =\frac{1}{B \rho} \frac{\partial B_{Y}}{\partial x}, \\
m & =\frac{1}{B \rho} \frac{\partial^{2} B_{Y}}{\partial x^{2}} .
\end{aligned}
$$

The difference of path length from that of the reference in $n$ revolutions, $\Delta L_{n}$, is calculated using the formula

$$
\Delta L_{n}=\int_{s_{0}}^{s_{0}+n C_{0}}\left[\sqrt{(1+h x)^{2}+x^{\prime 2}+y^{\prime 2}}-1\right] d s .
$$

Here, $C_{0}$ is the circumference of the reference orbit and $s_{0}$ is $s$ at the initial point. When terms higher than the second order are ignored, Eq. (4a) is approximated as

$$
\Delta L_{n}=\int_{s_{0}}^{s_{0}+n C_{0}}\left(h x+\frac{1}{2} x^{\prime 2}+\frac{1}{2} y^{\prime 2}\right) d s .
$$

This $\Delta L_{n}$ can be separated into an oscillating part and an increasing (or decreasing) part with $n$. The oscillating part spreads in the azimuthal direction and the increase (or decrease) shifts the average path length. Then the shift, $\Delta C$, is given by

$$
\Delta C=\lim _{n \rightarrow \infty} \frac{1}{n} \Delta L_{n} .
$$

This report aims to calculate $\Delta C$. The integral in Eq. (4b) will be evaluated in three parts: the $h x$ term using only linear $x$ motion, the second order terms $\frac{1}{2} x^{\prime 2}+\frac{1}{2} y^{\prime 2}$, and the $h x$ term from nonlinear $x$ motion.

\section{B. Contribution of linear part}

In this section, we review calculations with the first order approximation (linear approximation), the oscillating part of the integral, covered in Ref. [3] by the same author. The radial displacement produced by the linear betatron oscillation is written as

$$
x(s)=\sqrt{2 J_{X} \beta_{X}(s)} \sin \psi_{X}(s) .
$$

The total change of the path length after $n$ revolutions, $\Delta L_{n}$, is given by

$$
\begin{aligned}
\Delta L_{n}\left(s_{0}\right)= & \sqrt{2 J_{X}} \sum_{m=0}^{n-1} \int_{s_{0}}^{s_{0}+C_{0}}\left[h(s) \sqrt{\beta_{X}(s)}\right] \sin \left[\psi_{X}(s)\right. \\
& \left.+2 m \pi \nu_{X}\right] d s
\end{aligned}
$$

Here $\nu$ with the suffix " $X$ " or " $Y$ " is a betatron tune. In order to simplify Eq. (7), we use the following equations,

$$
\begin{aligned}
& \sum_{m=0}^{n-1} \sin [(2 m+1) \pi \nu+\psi]=\frac{\sin n \pi \nu}{\sin \pi \nu} \sin (n \pi \nu+\psi), \\
& \sum_{m=0}^{n-1} \cos [(2 m+1) \pi \nu+\psi]=\frac{\sin n \pi \nu}{\sin \pi \nu} \cos (n \pi \nu+\psi) .
\end{aligned}
$$

We also use dispersion function, $\eta(s)$, and dispersion angle, $\eta^{\prime}(s)$, given by

$$
\begin{aligned}
\eta\left(s_{0}\right) & =\frac{\sqrt{\beta_{X}\left(s_{0}\right)}}{2 \sin \pi \nu_{X}} \int_{s_{0}}^{s_{0}+C_{0}} h(s) \sqrt{\beta_{X}(s)} \cos \left[\psi_{X}(s)-\psi_{X}\left(s_{0}\right)-\pi \nu_{X}\right] d s, \\
\alpha_{X}\left(s_{0}\right) \eta\left(s_{0}\right)+\beta_{X}\left(s_{0}\right) \eta^{\prime}\left(s_{0}\right) & =\frac{\sqrt{\beta_{X}\left(s_{0}\right)}}{2 \sin \pi \nu_{X}} \int_{s_{0}}^{s_{0}+C_{0}} h(s) \sqrt{\beta_{X}(s)} \sin \left[\psi_{X}(s)-\psi_{X}\left(s_{0}\right)-\pi \nu_{X}\right] d s .
\end{aligned}
$$

Using these equations, Eq. (7) is simplified to [9]

$$
\begin{aligned}
\Delta L_{n}\left(s_{0}\right)= & \sqrt{2 J_{X} H\left(s_{0}\right)}\left\{-\cos \left[2 n \pi \nu_{X}+\psi_{X}\left(s_{0}\right)+\psi_{H}\left(s_{0}\right)\right]\right. \\
& \left.+\cos \left[\psi_{X}\left(s_{0}\right)+\psi_{H}\left(s_{0}\right)\right]\right\} .
\end{aligned}
$$

Here we used the functions $H(s)$ and $\psi_{H}(s)$ defined by

$$
\begin{aligned}
\sqrt{H} \sin \psi_{H} & =\eta\left(\alpha_{X} / \sqrt{\beta_{X}}\right)+\eta^{\prime} \sqrt{\beta_{X}}, \\
\sqrt{H} \cos \psi_{H} & =\eta / \sqrt{\beta_{X}} .
\end{aligned}
$$

The $H$ is the well-known function calculated by

$$
H=\gamma_{X} \eta^{2}+2 \alpha_{X} \eta \eta \prime+\beta_{X} \eta^{\prime 2} .
$$

$\Delta L_{n}$ has an oscillating part and not an increasing (or decreasing) part. Therefore the leading terms of $\Delta C$ are the second order terms.

\section{Contribution of trajectory slope terms}

We will calculate a part of the second order terms of the integration of Eq. (4b),

$$
\Delta L_{n, Y Y}=\int_{s_{0}}^{s_{0}+n C_{0}}\left(\frac{1}{2} y^{\prime 2}\right) d s
$$

Generally $y^{\prime}$ is expressed by the equation

$$
y^{\prime}(s)=\sqrt{2 J_{Y} \gamma_{Y}(s)} \sin \psi_{Y}(s)
$$


Substituting Eq. (14) into Eq. (4b) gives

$$
\begin{aligned}
\Delta L_{n, Y Y}\left(s_{0}\right)= & J_{Y} \sum_{m=0}^{n-1} \int_{s_{0}}^{s_{0}+C_{0}} \gamma_{Y}(s) \sin ^{2}\left[\psi_{Y}(s)+2 m \pi \nu_{Y}\right] d s \\
= & \frac{1}{2} J_{Y} \sum_{m=0}^{n-1} \int_{s_{0}}^{s_{0}+C_{0}} \gamma_{Y}(s)\left\{1-\cos \left[2 \psi_{Y}(s)\right.\right. \\
& \left.\left.+4 m \pi \nu_{Y}\right]\right\} d s .
\end{aligned}
$$

By dropping the oscillating part in Eq. (15), we obtain the contribution to the average path length as

$$
\lim _{n \rightarrow \infty} \frac{1}{n}\left[\int_{s_{0}}^{s_{0}+n C_{0}}\left(\frac{1}{2} y^{\prime 2}\right) d s\right]=\frac{1}{2} J_{Y} \int_{0}^{C_{0}} \gamma_{Y}(s) d s .
$$

Here we dropped $s_{0}$ because the integration is an invariant to $s_{0}$. The contribution of the $x^{\prime 2}$ term is calculated with the same way as

$$
\lim _{n \rightarrow \infty} \frac{1}{n}\left[\int_{s_{0}}^{s_{0}+n C_{0}}\left(\frac{1}{2} x^{\prime 2}\right) d s\right]=\frac{1}{2} J_{X} \int_{0}^{C_{0}} \gamma_{X}(s) d s .
$$

\section{Contribution of nonlinear $x$}

The additional displacement of $x$ to the linear $x$ is a sum of contributions of six nonlinear terms on the right-hand side of Eq. (2a). The contribution of each term can be calculated straightforwardly. As an example, we will calculate the contribution of $\frac{1}{2}\left(h^{\prime \prime}-h k-m\right) y^{2}$. Suppose that the ring has only one thin second order component in the linear lattice at $s=s_{0}$ and the horizontal deflection, $\Delta \theta_{X}$, by this thin component is given by

$$
\Delta \theta_{X}=\Delta \lambda y^{2} .
$$

Here $\Delta \lambda$ is a coefficient, that $\Delta \lambda=\frac{1}{2}\left(h^{\prime \prime}-h k-m\right) d s$ for this term. The vertical displacement $y$ is generally expressed as

$$
y(s)=\sqrt{2 J_{Y} \beta_{Y}(s)} \cos \psi_{Y}(s),
$$

and the horizontal beam deflection at the $n$th turn is

$$
\Delta \theta_{n, X}=2 \Delta \lambda J_{Y} \beta_{Y}\left(s_{0}\right) \cos ^{2}\left[\psi_{Y}\left(s_{0}\right)+2(n-1) \pi \nu_{Y}\right] .
$$

The nonlinear displacement is a sum of the smallperturbed betatron motion produced by $\Delta \theta_{n, X}$ in every turn and is written as a function of $s$ and $n$. The orbit displacement produced by a single deflection $\Delta \theta_{n, X}$ at the $p$ th turn, we will refer it by $\Delta x_{n}$, is given as

$$
\begin{aligned}
\Delta x_{n}= & \Delta \theta_{n, X} \sqrt{\beta_{X}\left(s_{0}\right) \beta_{X}(s)} \sin \left[\psi_{X}(s)-\psi_{X}\left(s_{0}\right)+2(p\right. \\
& \left.-n) \pi \nu_{X}\right] .
\end{aligned}
$$

The change of the path length from the $n$th to the $m$ th turn by $\Delta \theta_{n, X}$, is referred to as $\Delta L_{n, m}$, which is obtained as

$$
\begin{aligned}
\Delta L_{n, m}= & \Delta \theta_{n, X} \sqrt{\beta_{X}\left(s_{0}\right)} \sum_{p=n}^{m} \int_{s_{0}}^{s_{0}+C_{0}}\left[h(s) \sqrt{\beta_{X}(s)}\right] \\
& \times \sin \left[\psi_{X}(s)-\psi_{X}\left(s_{0}\right)+2(p-n) \pi \nu_{X}\right] d s .
\end{aligned}
$$

$\Delta L_{n, m}$ is simplified as we did for the linear term.

$$
\begin{aligned}
\Delta L_{n, m}= & \Delta \theta_{n, X} \sqrt{\beta_{X}\left(s_{0}\right) H\left(s_{0}\right)}\left\{\cos \psi_{H}\left(s_{0}\right)\right. \\
& \left.-\cos \left[2(m-n+1) \pi \nu_{X}+\psi_{H}\left(s_{0}\right)\right]\right\} .
\end{aligned}
$$

Dropping the oscillating term we obtain the change in the average path length for one revolution as

$$
\begin{aligned}
\lim _{m \rightarrow \infty} \frac{1}{m} \sum_{n=1}^{m} \Delta L_{n, m}= & 2 \Delta \lambda J_{Y} \beta_{Y}\left(s_{0}\right) \frac{1}{2} \\
& \times \sqrt{\beta_{X}\left(s_{0}\right) H\left(s_{0}\right)} \cos \psi_{H}\left(s_{0}\right) \\
= & \Delta \lambda J_{Y} \beta_{Y}\left(s_{0}\right) \eta\left(s_{0}\right) .
\end{aligned}
$$

Substitute $\Delta \lambda=\frac{1}{2}\left(h^{\prime \prime}-h k-m\right) d s$ into Eq. (24) and integrate it over the ring, we obtain the contribution to the shift as

$$
\frac{1}{2} J_{Y} \int_{0}^{C_{0}}\left(h^{\prime \prime}-h k-m\right) \eta \beta_{Y} d s .
$$

After the calculation of the contributions of the other five terms, we obtain the total shift as

$$
\begin{aligned}
\Delta C= & \frac{1}{2} J_{Y} \int_{0}^{C_{0}}\left[(1-h \eta) \gamma_{Y}+\left(h^{\prime \prime}-h k-m\right) \eta \beta_{Y}\right. \\
& \left.-2 h^{\prime} \eta \alpha_{Y}\right] d s+\frac{1}{2} J_{X} \int_{0}^{C_{0}}\left[(1+h \eta) \gamma_{X}\right. \\
& \left.+\left(4 h k-2 h^{3}+m\right) \eta \beta_{X}-2 h^{\prime} \eta \alpha_{X}\right] d s .
\end{aligned}
$$

The ways of deductions for the other terms are given in Appendix A.

\section{E. Relation to chromaticities}

The chromaticities are also obtained from Eqs. (2). Replacing $x$ in Eqs. (2) with $X+\eta \delta$ gives the following equations:

$$
\begin{aligned}
\eta^{\prime \prime}=h+\left(k-h^{2}\right) \eta & \\
{\left[\partial x^{\prime \prime} / \partial \delta\right]_{\delta=0}=} & {\left[\left(4 h k-2 h^{3}+m\right) \eta+h^{\prime} \eta^{\prime}+2 h^{2}-k\right] x } \\
& +(h \eta)^{\prime} x^{\prime} \\
{\left[\partial y^{\prime \prime} / \partial \delta\right]_{\delta=0}=} & {\left[-(m+2 h k) \eta-h^{\prime} \eta^{\prime}+k\right] y } \\
& +(h \eta)^{\prime} y^{\prime} .
\end{aligned}
$$

From Eqs. (28) we obtain the horizontal chromaticity, $\xi_{X}$, and the vertical chromaticity, $\xi_{Y}$, respectively. They are 


$$
\begin{aligned}
\xi_{X}= & \frac{1}{4 \pi} \int_{0}^{C_{0}}\left\{-\left[\left(4 h k-2 h^{3}+m\right) \eta+h^{\prime} \eta^{\prime}\right.\right. \\
& \left.\left.+2 h^{2}-k\right] \beta_{X}+(h \eta)^{\prime} \alpha_{X}\right\} d s, \\
\xi_{Y}= & \frac{1}{4 \pi} \int_{0}^{C_{0}}\left\{\left[(m+2 h k) \eta+h^{\prime} \eta^{\prime}-k\right] \beta_{Y}\right. \\
& \left.+(h \eta)^{\prime} \alpha_{Y}\right\} d s .
\end{aligned}
$$

These are consistent with the results solved under more limited conditions [10-12]. Equations (29) have many equivalent forms and can be reformed to the following equations:

$$
\begin{aligned}
\xi_{X}= & \frac{1}{4 \pi} \int_{0}^{C_{0}}\left[-(1+h \eta) \gamma_{X}\right. \\
& \left.-\left(4 h k-2 h^{3}+m\right) \eta \beta_{X}+2 h^{\prime} \eta \alpha_{X}\right] d s \\
\xi_{Y}= & \frac{1}{4 \pi} \int_{0}^{C_{0}}\left[(-1+h \eta) \gamma_{Y}\right. \\
& \left.-\left(h^{\prime \prime}-h k-m\right) \eta \beta_{Y}+2 h^{\prime} \eta \alpha_{Y}\right] d s
\end{aligned}
$$

We used equations listed in Appendix B for the reformation. Using Eqs. (30), Eq. (26) is simplified to

$$
\Delta C=-2 \pi\left(J_{X} \xi_{X}+J_{Y} \xi_{Y}\right) .
$$

\section{EFFECT OF THE CHANGE OF PATH LENGTH}

In an electron storage ring, an rf system maintains a fixed path length for all trajectories, thus the rf system counteracts the path lengthening by changing the electron momentum. The shift of momentum, $\delta$, to cancel the shift of path length, $\Delta C$, is given by

$$
\delta=-\frac{1}{\alpha} \frac{\Delta C}{C_{0}} .
$$

The momentum shift causes a shift in the synchronous rf phase $\left(\phi_{S}\right)$ where the energy loss per revolution and energy gain by the acceleration are balanced. From now on, we assume that $J_{Y}=0$ to simplify the calculation. When $\phi_{S}$ of the reference electron, $\phi_{S 0}$, is given by the equation

$$
e V_{\mathrm{rf}} \sin \phi_{S 0}=U_{0},
$$

the shift of the synchronous phase, $\Delta \phi_{S}$, is given by

$$
\left(1+\kappa_{\mathrm{rf}} J_{X}\right) e V_{\mathrm{rf}} \sin \left(\phi_{S 0}+\Delta \phi_{S}\right)=U_{0}\left(1+J_{\varepsilon} \delta+J_{\beta} J_{X}\right) .
$$

Here, $e, V_{\mathrm{rf}}$, and $U_{0}$ are elementary charge, rf acceleration voltage, and the radiation loss per revolution of the reference particle, respectively. $J_{\epsilon}$ is the damping partition number. $J_{\beta}$ and $\kappa_{\text {rf }}$ are factors that represent the dependences of the radiation loss and energy gain on $J_{X}$. From Eq. (34) we obtain $\Delta \phi_{S}$ as

$$
\Delta \phi_{S}=-\tan \phi_{S 0}\left(\kappa_{\mathrm{rf}}+\frac{J_{\varepsilon}}{\alpha} \frac{2 \pi \xi_{X}}{C_{0}}+J_{\beta}\right) J_{X} .
$$

In most storage rings $\kappa_{\mathrm{rf}}$ and $J_{\beta}$ are negligible and Eq. (35) is approximated by

$$
\Delta \phi_{S}=-\frac{J_{\varepsilon}}{\alpha} \frac{2 \pi \xi_{X} J_{X}}{C_{0}} \tan \phi_{S} .
$$

The above shift creates a bunch spread given by $\Delta \phi_{S} /\left(2 \pi f_{\mathrm{rf}}\right)$. Here $f_{\mathrm{rf}}$ is the rf frequency of the accelerating cavity.

This consequence is very much different from that of the linear oscillating term [3]. The linear oscillating term is obtained from Eq. (10) as

$$
\Delta L_{n}\left(s_{0}\right)=-\sqrt{2 J_{X} H\left(s_{0}\right)} \cos \left[2 n \pi \nu_{X}+\psi_{X}\left(s_{0}\right)+\psi_{H}\left(s_{0}\right)\right] .
$$

On the other hand, from Eq. (6), the betatron motion at $s=s_{0}$ is given by

$$
x\left(s_{0}\right)=\sqrt{2 J_{X} \beta_{X}\left(s_{0}\right)} \sin \left[2 n \pi \nu+\psi_{X}(s)\right] .
$$

\begin{tabular}{|c|c|}
\hline Parameters & Quantities \\
\hline Stored electron energy: $E$ & $1.0 \mathrm{GeV}$ \\
\hline Circumference: $C_{0}$ & $118.7 \mathrm{~m}$ \\
\hline Curvature of radius: $1 / h$ Normal bend & $3.217 \mathrm{~m}$ \\
\hline Inverse bend & $-3.201 \mathrm{~m}$ \\
\hline Horizontal chromaticity: $\xi_{X}$ & -7 \\
\hline Damping partition number $J_{\varepsilon}$ & 2.01 \\
\hline rf frequency: $f_{\mathrm{rf}}$ & $499.956 \mathrm{MHz}$ \\
\hline rf acceleration voltage: $V_{\mathrm{rf}}$ & $300 \mathrm{kV}$ \\
\hline rf synchronous phase: $\phi_{S}$ & $0.116 \mathrm{rad}$ \\
\hline Linear momentum compaction factor: $\alpha_{1}$ & $2 \times 10^{-6}$ \\
\hline 3rd order momentum compaction factor: $\alpha_{3}$ & 0.9 \\
\hline Natural emittance: $\varepsilon_{X N}$ & $30.1 \mathrm{~nm}$ \\
\hline Natural energy spread: $\sigma_{E N}$ & $0.047 \%$ \\
\hline Natural bunch length: $\sigma_{T}$ & $0.42 \mathrm{ps}$ \\
\hline
\end{tabular}

These equations show that a particle moves on an ellipse in $s-x$ plane. The amplitude of the oscillation in azimuthal direction is $\sqrt{2 J_{X} H\left(s_{0}\right)}$, which has no explicit dependence on $\alpha$. This linear spread depends on the location of light source point $\left(s_{0}\right)$ and is zero at the dispersion free sections. The other difference is that the linear effect appears as fast as the betatron motion. It spreads the beam in one revolution.

\section{CALCULATIONS AT NEWSUBARU}

In this section we calculate shifts of some parameters for one electron with finite $J_{X}$ using ring parameters from an existing QI ring, NewSUBARU. Table I lists the ring parameters when operated in the extreme QI mode [13].

TABLE I. Main parameters of NewSUBARU at QI condition. 


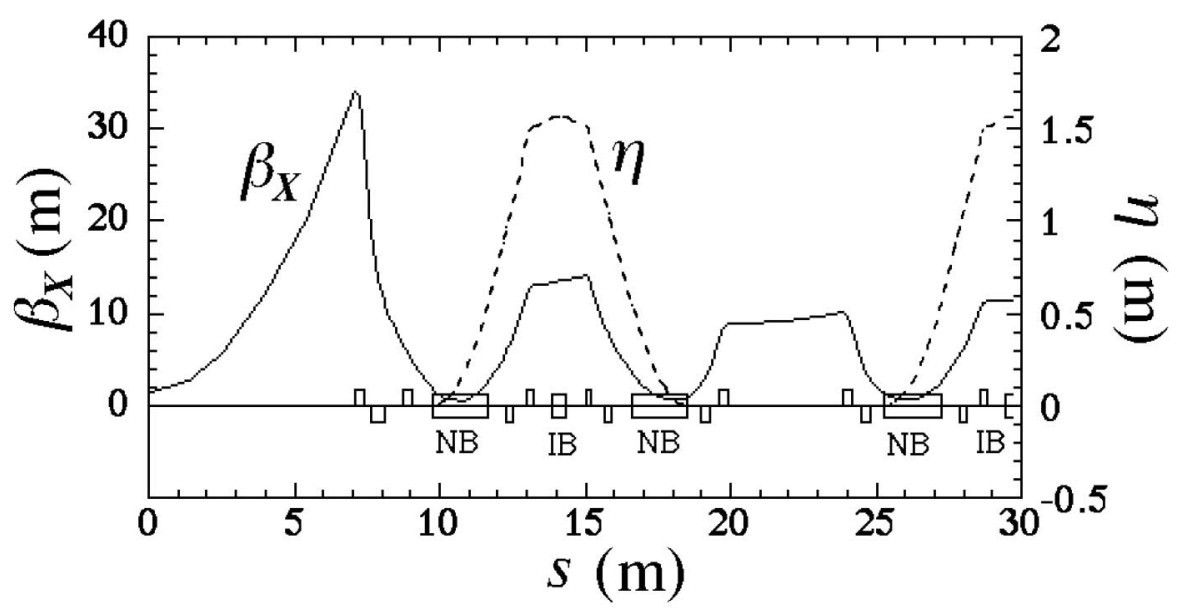

FIG. 1. Beta function $\left(\beta_{X}\right.$, solid line) and dispersion function $(\eta$, broken line) in $1 / 4$ of NewSUBARU. The boxes indicate the locations and lengths of the bending magnets (indicated by NB and IB) and quadrupole magnets.

Figure 1 shows the horizontal beta function, $\beta_{X}$, and the dispersion function, $\eta$, in $1 / 4$ of the ring.

For $J_{X}$ as a typical value, we will use a half of the natural emittance, $\varepsilon_{X N} / 2 . \Delta C / C_{0}$ is calculated to be $5.6 \times 10^{-9}$. For this electron, $\delta$ is calculated to be $-0.28 \%$, which is much larger than the rms of the natural energy spread, $0.047 \%$. This suggests that the horizontal chromaticity and the longitudinal chromaticity ( $\delta$ dependence of the synchrotron tune) must be controlled in case of extreme bunch shortening. The phase shift, $\Delta \phi_{S}$, is calculated to be $6.5 \times 10^{-4}$ radian $(0.21 \mathrm{ps}$ in time), which is smaller than the natural bunch length, $0.42 \mathrm{ps}$. Thus, we conclude that the momentum spread would be considerably large, but the bunch lengthening would not.

However, in a real machine the above conclusion should be modified to consider the effect of the higher order momentum compaction factor. Then $\Delta C / C_{0}$ is written as

$$
\Delta C / C_{0}=\alpha_{1} \delta+\alpha_{2} \delta^{2}+\alpha_{3} \delta^{3}+,
$$

where $\alpha_{n}$ is $n$th order momentum compaction factor. It is known that $\alpha_{2}$ of the QI ring should be adjusted to zero. Then the leading nonlinear term is $\alpha_{3}$. We ignore terms higher than third order then the above equation is approximated as

$$
\Delta C / C_{0}=\alpha_{1} \delta+\alpha_{3} \delta^{3} .
$$

Using Eq. (40) instead of Eq. (32), $\delta$ is calculated to be $-0.14 \%$. On the other hand the off-momentum linear momentum compaction factor is given by [14]

$$
\alpha=\frac{d}{d \delta}\left(\frac{\Delta C}{C}\right) \approx \alpha_{1}+3 \alpha_{3} \delta^{2} .
$$

The $\alpha$ for the electron with is $J_{X}=\varepsilon_{X N} / 2$ is $7.6 \times$ $10^{-6}$, which is about 4 times of the $\alpha_{1}$. This means that the expected bunch lengthening by the enlarged $\alpha$ is con- siderable when $\alpha_{3}$ is large. The bunch lengthening by the linear oscillation is calculated to be about $0.2 \mathrm{ps}$ at the light source point for the bunch length measurement. Both of the effects of the linear and the second order should be considered to realize a sub-ps short bunch.

\section{ACKNOWLEDGMENTS}

The author thanks Dr. G. Wüestefeld of BESSY for his important suggestion and fruitful discussions on this subject. Dr. T. Koseki of RIKEN offered a field map of the rf cavity, which was used to confirm that $k_{\mathrm{rf}}$ was negligible in the extreme case.

\section{APPENDIX A: CONTRIBUTIONS OF SECOND ORDER TERMS TO AVERAGE PATH LENGTH}

In the process of the deduction of Eq. (26), we calculate the contributions to $\Delta C$ of the six nonlinear terms of Eq. (2a). A deduction of the contribution of one term is given in the main text. The term calculated in the main text has the form of Eq. (18), $\Delta \theta_{X}=\Delta \lambda y^{2}$. The result before the integration is given by Eq. (26) as $\Delta \lambda J_{Y} \beta_{Y} \eta$. In this appendix, ways of deduction for the other five terms are given.

A contribution of the term of the form $\Delta \theta_{X}=\Delta \lambda x^{2}$ is calculated with the same way by using the equation $x=$ $\sqrt{2 J_{X} \bar{\beta}_{X}} \cos \psi_{X}$ instead of Eq. (19), $y=\sqrt{2 J_{Y} \bar{\beta}_{Y}} \cos \psi_{Y}$. The equations to the result, from Eq. (21)-(23), remain the same. Then with replacements of $J_{Y}$ and $\beta_{Y}$ with $J_{X}$ and $\beta_{X}$, Eq. (24) gives a correct result for this term. The result before the integration is $\Delta \lambda J_{X} \beta_{X} \eta$.

A contribution of the term of the form $\Delta \theta_{X}=\Delta \lambda y^{\prime 2}$ is calculated by using $y^{\prime}=\sqrt{2 J_{Y} \gamma_{Y}} \cos \psi_{Y}$ instead of Eq. (19). The result is obtained by a replacement of $\beta_{Y}$ with $\gamma_{Y}$ as $\Delta \lambda J_{Y} \gamma_{Y} \eta$. For the term of the form $\Delta \theta_{X}=$ $\Delta \lambda x^{\prime 2}$ we use $x^{\prime}=\sqrt{2 J_{X} \gamma_{X}} \cos \psi_{X}$ instead of Eq. (19) and obtain $\Delta \lambda J_{X} \gamma_{X} \eta$. 
For the term of the form $\Delta \theta_{X}=\Delta \lambda y y^{\prime}$ we use the following two equations,

$$
\begin{gathered}
y(s)=\sqrt{\varepsilon_{Y} \beta_{Y}(s)} \cos \psi_{Y}(s), \\
y^{\prime}(s)=-\sqrt{\varepsilon_{Y} / \beta_{Y}(s)}\left[\alpha_{Y} \cos \psi_{Y}(s)+\sin \psi_{Y}(s)\right] .
\end{gathered}
$$

The horizontal beam deflection at the $n$th turn is

$$
\begin{aligned}
\Delta \theta_{n, X}= & -\Delta \lambda J_{Y}\left\{2 \alpha_{Y}\left(s_{0}\right) \cos ^{2}\left[\psi_{Y}\left(s_{0}\right)+2(n-1) \pi \nu_{Y}\right]\right. \\
& \left.+\sin \left[2 \psi_{Y}\left(s_{0}\right)+4(n-1) \pi \nu_{Y}\right]\right\} .
\end{aligned}
$$

Since the second term in the bracket \{\} is oscillatory, it can be dropped and that equation becomes the same as Eq. (20) with the replacement of $-\alpha_{Y}$ with $\beta_{Y}$. Then the result before the integration is $-\Delta \lambda J_{Y} \alpha_{Y} \eta$. For the term of the form $\Delta \theta_{X}=\Delta \lambda x x^{\prime}$, we obtain $-\Delta \lambda J_{X} \alpha_{X} \eta$ by the similar replacements of $J_{Y}$ and $a_{Y}$ in the former result with $J_{X}$ and $\alpha_{X}$.

Summing up results for these six terms with substitutions of appropriate coefficient terms to $\Delta \lambda$ makes the equation,

$$
\begin{aligned}
\lim _{m \rightarrow \infty} \frac{1}{m} \sum_{n=1}^{m} \Delta L_{n, m}= & {\left[\frac{1}{2}\left(h^{\prime \prime}-h k-m\right) d s\right] J_{Y} \eta \beta_{Y} } \\
& +\left[\left(2 h k-h^{3}+\frac{1}{2} m\right) d s\right] J_{X} \eta \beta_{X} \\
& -\left(\frac{1}{2} h d s\right) J_{Y} \eta \gamma_{Y}+\left(\frac{1}{2} h d s\right) J_{X} \eta \gamma_{X} \\
& -\left(h^{\prime} d s\right) J_{Y} \eta \alpha_{Y}-\left(h^{\prime} d s\right) J_{X} \eta \alpha_{X} .
\end{aligned}
$$

Equation (26) is obtained by the integration of Eq. (A4) over the ring and addition of Eqs. (16) and (17).

\section{APPENDIX B: USEFUL FORMULAS FOR REFORMATION}

The equations for chromaticities, Eqs. (29), and the shift, Eq. (26), have many equivalent forms. This appendix gives useful formulas for equivalent reformations.

The magnetic field parameter $h$, Twiss parameters $\alpha_{X}$, $a_{Y}, \beta_{X}$, and $\beta_{Y}$, dispersion function $\eta$ and the differentiations of them to $s$ are unique functions of $s$. This means that the functions are closed in the ring. Then any products of any of these functions should be a closed function. It is obvious that the integration over the ring of a differentiation of a closed function should be zero. Then we have following equations,

$$
\begin{aligned}
& \int_{0}^{C_{0}}\left(h \eta \alpha_{X}\right)^{\prime} d s=0, \\
& \int_{0}^{C_{0}}\left(h \eta \alpha_{Y}\right)^{\prime} d s=0, \\
& \int_{0}^{C_{0}}\left(h \eta^{\prime} \beta_{X}\right)^{\prime} d s=0, \\
& \int_{0}^{C_{0}}\left(h^{\prime} \eta \beta_{Y}\right)^{\prime} d s=0 .
\end{aligned}
$$

Expansion of the functions in the integrals gives the following equations:

$$
\begin{array}{r}
\int_{0}^{C_{0}}\left[(h \eta)^{\prime} \alpha_{X}+(h \eta)\left(h^{2}-k\right) \beta_{X}-(h \eta) \gamma_{X}\right] d s=0, \\
\int_{0}^{C_{0}}\left[(h \eta)^{\prime} \alpha_{Y}+(h \eta)\left(k \beta_{Y}-\gamma_{Y}\right)\right] d s=0, \\
\int_{0}^{C_{0}}\left(h^{\prime} \eta^{\prime} \beta_{X}-2 h \eta^{\prime} \alpha_{X}+h \eta^{\prime \prime} \beta_{X}\right) d s=0 \\
\int_{0}^{C_{0}}\left(h^{\prime \prime} \eta \beta_{Y}+h^{\prime} \eta^{\prime} \beta_{Y}-2 h^{\prime} \eta \alpha_{Y}\right) d s=0
\end{array}
$$

We also use the following equations of Twiss parameters,

$$
\begin{aligned}
& \gamma_{X}=\left(h^{2}-k\right) \beta_{X}-\alpha_{X}^{\prime}, \\
& \gamma_{Y}=k \beta_{Y}-\alpha_{Y}^{\prime} .
\end{aligned}
$$

Substituting Eq. (27) into $\eta^{\prime \prime}$ of Eq. (B2c) and using Eq. (B2a), we obtain

$$
\int_{0}^{C_{0}}\left[\left(h^{\prime} \eta^{\prime}+h^{2}\right) \beta_{X}+\left(h^{\prime} \eta-h \eta^{\prime}\right) \alpha_{X}-h \eta \gamma_{X}\right] d s=0 .
$$

These equations were used to reform Eqs. (29). For example, Eq. (B2d) was used to eliminate the integration of $h^{\prime} \eta^{\prime} \beta_{Y}$ from Eqs. (29b) and (B4) was used to eliminate the integration of $h^{\prime} \eta^{\prime} \beta_{X}$ from Eq. (29a).

[1] M. Abo-Bakr, J. Feikes, K. Holldack, G. Wüstefeld, and H.-W. Hübers, Phys. Rev. Lett. 88, 254801 (2002).

[2] F. Sannibale, J. M. Byrd, Á. Loftsdóttir, M. Venturini, M. Abo-Bakr, J. Feikes, K. Holldack, P. Kuske, G. Wüstefeld, H.-W. Hübers, and R. Warnock, Phys. Rev. Lett. 93, 094801 (2004).

[3] Y. Shoji, Phys. Rev. ST Accel. Beams 7, 090703 (2004).

[4] H. Wiedemann, Particle Accelerator Physics II (SpringerVerlag, Berlin, 1995), Chap. 6, p. 208.

[5] L. Emery, in Proceedings of 15th International Conference on High Momentum Accelerators, edited by J. Rossbach (World Scientific, Singapole, 1993), p. 1172. 
[6] É. Forest, Beam Dynamics-A New Attitude and Framework (Harwood Academic Publishers, Amsterdam, 1998), Chap. 9, p. 264.

[7] J. Murphy, Report No. BNL42333, 1996, p. 5.

[8] H. Wiedemann, Particle Accelerator Physics II (SpringerVerlag, Berlin, 1995), Chap. 4, p. 94.

[9] A. Piwinski, and A. Wrulich, Report No. DESY 76/71, 1976; A. Piwinski, Handbook of Accelerator Physics and Engineering, edited by A. Wu Chao and M. Tigner (World Scientific, Singapore, 1999), Chap. 2, p. 72.

[10] F. Willeke and G. Ripken, in Physics of Particle Accelerators, edited by Melvin Month and Margaret Dienes, AIP Conf. Proc. No. 184 (AIP, New York, 1987), p. 808.
[11] S. Guiducci, CAS1992, CERN94-01, p. 514.

[12] M. Takao, H. Tanaka, K. Soutome, and J. Schimizu, Phys. Rev. E 70, 016501 (2004).

[13] Y. Shoji, A. Ando, and S. Hashimoto, in Synchrotron Radiation Instrumentation, edited by $\mathrm{T}$. Warwick, J. Arthur, H. A. Padmore, and J. Stöhr, AIP Conf. Proc. No. 705 (AIP, New York, 2004), p. 57; Y. Shoji, S. Hisao, and T. Matsubara, in Proceedings of European Particle Accelerator Conference, Lucerne, 2004 (EPSAG, Lucerne, 2004), p. 2356.

[14] J. Feikes and G. Wüstefeld, in Proceedings of Particle Accelerator Conference, New York, 1999 (IEEE, Piscataway, NJ, 1999), p. 2376. 